\title{
HIGH RESOLUTION SPECTROSCOPY \\ AT THE ESO 50 CM TELESCOPE: \\ SPECTROSCOPIC MONITORING OF LUMINOUS HOT \\ STARS
}

\author{
A. KAUFER, H. MANDEL, O. STAHL, B. WOLF and \\ TH. SZEIFERT, TH. GÄNG, C.A. GUMMERSBACH \\ Landessternwarte Heidelberg-Königstuhl, D-69117 Heidelberg, Germany \\ and \\ J. KOVACS \\ Gothard Astrophysical Observatory, H-9707 Szombathely, Hungary
}

\begin{abstract}
Galactic Luminous Blue Variables and A- and B-type supergiants were monitored spectroscopically with high resolution in wavelength and time. Line profile variations on different timescales are found.
\end{abstract}

\section{Observations}

With the portable fiber-linked echelle spectrograph of the Landessternwarte Heidelberg-Königstuhl FraSH (Mandel, 1988) attached to the ESO $50 \mathrm{~cm}$ telescope at La Silla we monitored from February through May 1993 a few galactic Luminous Blue Variables (LBV) and several A- and B-type supergiants (cf. Wolf et al. 1993) at high resolution both in wavelength

TABLE I

List of monitored objects

\begin{tabular}{lll}
\hline object & sp & spectra/nights \\
\hline$\eta$ Car & pec (LBV) & $103 / 117$ \\
$\theta^{1}$ Ori C & O7 V & $78 / 89$ \\
AG Car & Ofpe-A2 (LBV) & $91 / 115$ \\
$\zeta^{1}$ Sco & B1 Ia-O & $93 / 114$ \\
$\beta$ Ori & B8 Ia & $86 / 104$ \\
HD 96919 & B9 Ia & $84 / 115$ \\
HD 92207 & A0 Ia & $86 / 115$ \\
HD 160529 & A2 Ia-O (LBV) & $71 / 101$ \\
\hline
\end{tabular}

$(R=20000)$ and time (each night one spectrum per object). With our EEV CCD with $1152 \times 770$ pixel of $22 \mu$ we cover $2700 \AA$ in one exposure (standard setup: $4050-6780 \AA)$. 


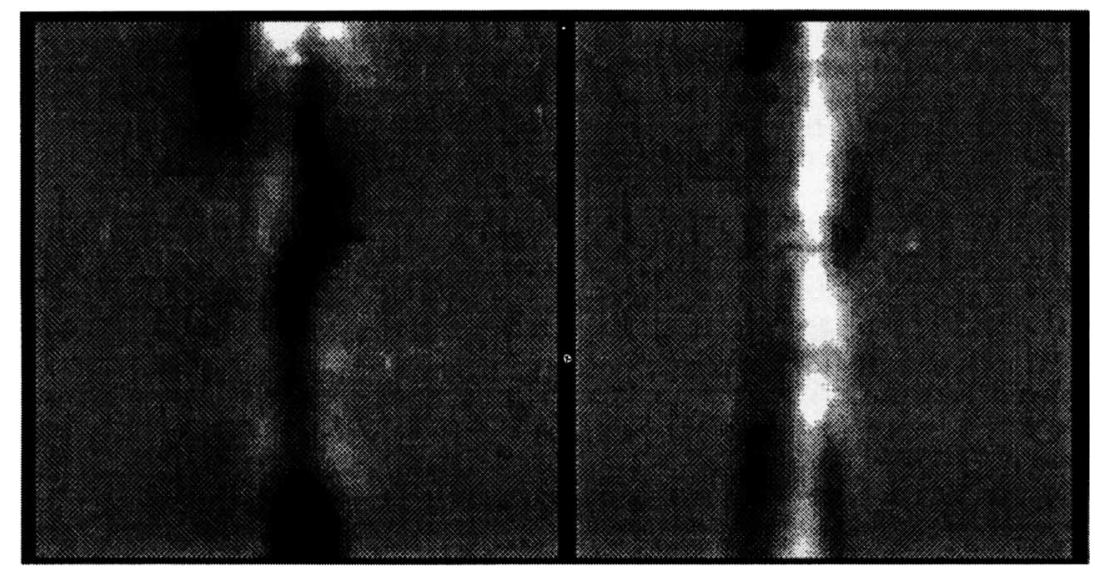

Fig. 1. Line profile variations of HeI 6678 (left) and Si II 6347 of AG Car. The lines are centered to the systemic velocity $(+20 \mathrm{~km} / \mathrm{s})$ of AG Car. The complete width of the abscissa is from -300 to $+300 \mathrm{~km} / \mathrm{s}$ for both lines. The ordinate covers the timespan from JD 2449023(bottom) to JD 2449139(top).

\section{Aims and first results}

The aim of the campaign is to study the time and depth dependent atmospheric velocity fields of these objects and the mass-loss variations derived from the $\mathrm{H} \alpha$-profile variations.

All monitored objects show line profile variations from strictly periodic as found for $\theta^{1}$ Ori C (cf. Stahl et al. 1993) to highly irregular as for $\beta$ Ori. The observed typical timescales of these variations due to the hydrodynamic processes in the extended atmospheres of these luminous objects range from a few days to a few weeks. Therefore daily observations over months are the right choice to monitor these stars.

\section{References}

Mandel, H.: 1988, in IAU Symp. 132, eds. G. Cayrel de Strobel and M. Spite, pg. 9-13, Kluwer.

Stahl, O., Wolf, B., Gäng, Th., Gummersbach, C.A., Kaufer, A., Kovacs, J., Mandel, H., Szeifert, Th.: 1993, A\&A, 274, L29-L32

Wolf, B., Mandel, H., Stahl, O., Kaufer, A., Gäng, Th., Gummersbach, C.A., Szeifert, Th.: 1993, "High resolution spectroscopy at the ESO $50 \mathrm{~cm}$ telescope": The ESO Messenger (submitted) 